\title{
Pénurie d'enseignants et difficultés dans l'enseignement de base à Shanghai
}

Shortage of teachers and difficulties in basic education in Shanghai

Penuria de docentes y dificultades en la enseñanza de base en Shanghái

Ren Jie

Traducteur : Sylvaine Herold

\section{(2) OpenEdition}

Journals

Édition électronique

URL : https://journals.openedition.org/ries/5816

DOI : $10.4000 /$ ries.5816

ISSN : 2261-4265

Éditeur

France Education international

Édition imprimée

Date de publication : 1 avril 2017

Pagination : 95-104

ISBN : 978-2-85420-614-2

ISSN : 1254-4590

Référence électronique

Ren Jie, "Pénurie d'enseignants et difficultés dans l'enseignement de base à Shanghai ", Revue internationale d'éducation de Sèvres [En ligne], 74 | avril 2017, mis en ligne le 01 avril 2019, consulté le 11 mars 2022. URL : http://journals.openedition.org/ries/5816 ; DOI : https://doi.org/10.4000/ries. 5816 


\title{
Pénurie d'enseignants et difficultés dans l'enseignement de base à Shanghai*
}

\author{
Ren Jie \\ Shanghai Teacher Training Center
}

En Chine, la formation initiale des nouveaux enseignants est actuellement le principal point de vigilance relatif au développement professionnel des enseignants. Cet article s'intéresse aux raisons et aux résultats des changements apportés à la formation initiale des enseignants en Chine.

\section{UNE PÉNURIE D’ENSEIGNANTS \\ Un taux d'abandon élevé des nouveaux enseignants}

Ces dernières années, la pénurie de personnel enseignant s'est en effet accrue rapidement en Chine. Si l'on considère l'exemple de Shanghai, le nombre de postes d'enseignants restés vacants a atteint 6000 en 2016 en ce qui concerne les écoles primaires, secondaires et maternelles, dont $60 \%$ dans les écoles primaires et maternelles. Il est donc urgent de résoudre le problème de pénurie d'enseignants.

Le développement rapide de l'économie chinoise et l'investissement croissant des Chinois dans l'éducation ont fait apparaître le besoin urgent d'amélioration et de développement de l'école en Chine. En tant que métropole internationale, Shanghai a attiré ces dernières années un nombre toujours plus grand de talents extérieurs et n'a eu de cesse d'assouplir les exigences d'accès à une école de proximité pour les enfants des travailleurs migrants. Ainsi, confrontée à une densité de population croissante et à une demande d'expansion urbaine toujours plus pressante, Shanghai a prévu de créer plus de 800 établissements d'enseignement de base d'ici 2020, afin de soulager la pression sur des ressources éducatives déjà insuffisantes.

La Chine a en outre assoupli en 2015 sa politique de contrôle des naissances. On a ainsi observé en 2016 une hausse mensuelle du taux de natalité lié au deuxième enfant par famille, une tendance qui devrait se poursuivre jusqu'en 2018 et conduira à un taux de natalité tel que la situation de stress au moment de l'entrée à l'école n'en sera que plus nette. Dans le même temps, de nombreux jeunes enseignants abandonnent le métier.

* Article traduit par Sylvaine Herold. 


\section{Une importante pression concurrentielle}

À Shanghai, on a constaté que le taux d'abandon des enseignants débutants est le plus important dans les écoles privées, dans lesquelles ils perçoivent des salaires relativement bas pour une charge de travail élevée ainsi qu'une mise en concurrence intense en raison de la demande des écoles pour une plus grande compétitivité éducative. Les nouveaux enseignants travaillant dans ce type d'écoles ont à affronter cette situation concurrentielle, tout en bénéficiant d'un faible niveau de garantie de retraite, de sécurité de l'emploi et avec peu de perspectives de développement professionnel et de soutien apporté par ces écoles à la formation continue.

\section{Une pression au travail et un conflit entre travail et apprentissage}

En Chine, la carrière d'enseignant est peu sûre, y compris pour les nouveaux enseignants des écoles publiques, qui bénéficient pourtant d'un poste fixe, d'un taux de formation élevé et d'une planification normée de leur développement professionnel. En Chine, un professeur se qualifie d'abord en obtenant l'examen de certification nationale pour enseigner ${ }^{1}$ et la qualification correspondante, puis en suivant une formation en stage d'un an, organisée par les établissements nationaux de formation des enseignants et les "écoles d'affectation". Comparé à d'autres professions, le début de carrière d'un enseignant n'est donc pas de tout repos. Outre l'accomplissement des missions assignées par l'État, les nouveaux enseignants travaillant à Shanghai pour la première année doivent suivre une formation en «immersion " dans les «écoles de base pour le développement professionnel $»^{2}$, mais également une formation standardisée dispensée par les instituts de formation au niveau du district ou du comté. À cette fin, la Commission de l'éducation de Shanghai a stipulé en des termes explicites que les écoles d'affectation doivent réduire la charge de travail des nouveaux enseignants, mais cela est difficile à mettre en ouvre dans ces écoles pour des raisons liées au rendement du travail, à la pénurie de personnel, etc. Ainsi, l'importance du conflit entre travail et apprentissage pousse les nouveaux enseignants à terminer en hâte leur formation dans les écoles de base et à réaliser des heures supplémentaires pour satisfaire à leurs obligations professionnelles. 


\section{Des salaires sans rapport avec l'intensité du travail}

À Shanghai, le salaire moyen des enseignants de première et de deuxième année du secondaire inférieur est d'environ 4740 yuans $^{3}$, et il est encore plus bas pour les enseignants des écoles primaires et maternelles. Ce niveau de salaire est néanmoins bien plus élevé que le salaire minimum à Shanghai, qui est de 2190 yuans $^{4}$. Cependant la difficulté d'accès à la profession enseignante à Shanghai vient contrebalancer voire annuler l'avantage perçu du niveau de revenu des enseignants. Ce coût d'entrée élevé est également mis en évidence par le fait que les enseignants, y compris ceux de maternelle doivent détenir un diplôme de l'enseignement supérieur, de type Bachelor ou plus, ainsi que des qualifications de spécialité adaptées. L'enseignant doit en outre s'être enregistré à Shanghai avec sa famille afin de pouvoir exercer dans les écoles et jardins d'enfants publics, ce qui contraint bon nombre de candidats non locaux de niveau master à exercer dans des écoles privées de la périphérie aux exigences d'enregistrement plus floues. Ainsi, il n'est pas si aberrant, y compris pour des candidats de niveau doctorat, d'accepter d'exercer comme enseignants dans des écoles privées de niveau secondaire inférieur. Et bien que les nouveaux enseignants hautement qualifiés commencent à profiter à Shanghai de certains bénéfices et de certaines politiques préférentielles dans le système d'évaluation des titres professionnels, le système d'ancienneté continue de peser lourdement sur leurs possibilités de promotion, tant en termes de titres professionnels que de postes. Ainsi, après avoir supporté des débuts de carrière incertains, beaucoup de nouveaux enseignants, conscients d'un niveau de salaire et d'un statut social déséquilibrés par rapport au niveau d'éducation élevé requis et à l'intensité du travail, quittent définitivement la profession pour d'autres métiers, dans la perspective de salaires plus élevés et d'un développement professionnel plus avantageux.

\section{Des disciplines mineures et des enseignants hommes déconsidérés}

En Chine, l'accent est mis principalement sur les disciplines dites majeures, telles que le chinois, les mathématiques, les langues étrangères, la physique et la chimie. Les enseignants de ces matières bénéficient d'un statut plus élevé dans les écoles, phénomène qui tire son origine de l'importance excessive accordée par les parents à la qualité de l'enseignement scolaire. Ceux-ci considèrent de surcroît les résultats scolaires comme le principal critère 
d'évaluation de l'enseignement, notamment les résultats aux examens d'entrée au collège, au lycée et à l'université. Inévitablement, certaines écoles favorisent dès lors les enseignants des disciplines majeures, que ce soit en termes de crédits temps, d'organisation du programme, de recherche pédagogique, de formation ou d'évolution professionnelle, tout simplement parce que ces matières sont affectées de coefficients plus élevés dans les examens d'entrée cruciaux mentionnés précédemment. Cela est particulièrement visible dans les systèmes de rémunération liée à la performance, dans lesquels une attention insuffisante est accordée aux enseignants des disciplines "non examinées ", qui concourent pourtant à l'amélioration de la qualité de l'enseignement, telles que la musique, les arts, l'éducation physique et les compétences professionnelles - cependant que ces matières dites "mineures" font l'objet d'une attention renouvelée dans les établissements d'enseignement privés à but lucratif à l'extérieur des campus. Ainsi, d'un côté, les parents chinois attendent de l'école qu'elle se concentre sur l'enseignement des "matières majeures » afin d'améliorer la capacité de leurs enfants à passer des examens ; mais, de l'autre, ils sont disposés à verser d'importantes sommes d'argent à des établissements d'enseignement privés, afin de renforcer les aptitudes de leurs enfants en musique, arts, sports, etc. Dans un tel contexte, l'abandon des enseignants de ces matières mineures est un phénomène courant, et encore plus prononcé chez les nouveaux enseignants.

La situation en matière de développement professionnel est encore plus difficile en Chine pour les enseignants de sexe masculin, en particulier ceux travaillant dans les écoles maternelles et primaires. D’une part, sous l'influence d'un chauvinisme masculin séculaire, les Chinois attendent des hommes un statut social relativement plus élevé que celui des femmes - dans une société où l'idée que «tout le monde est un homme d'affaires » et où la référence à la «norme bureaucratique " sont très présentes - alors que les enseignants de sexe masculin n'ont de fait aucun avantage en termes de revenu et de statut social. D'autre part, les parents chinois accordent une importance excessive à leur enfant unique, ce qui fait qu'au niveau maternel et élémentaire, les enseignantes sont plus populaires que les enseignants car réputées plus patientes et plus attentionnées que leurs collègues masculins. Les enseignants de sexe masculin sont donc peu nombreux dans les écoles maternelles et primaires, et cela représente également un facteur important d'abandon du personnel enseignant de sexe masculin en période de stage.

\section{DES EFFETS SUR LA FORMATION}

Dans le même temps, de plus en plus de diplômés non normaliens admis au test de qualification des enseignants sont entrés dans la profession enseignante, ce qui modifie la composition de la population des enseignants débutants. De ce fait, le modèle traditionnel de tutorat se trouve dans l'impossibilité de répondre à la demande croissante de formation des nouveaux enseignants, tant en termes quantitatifs que qualitatifs. 


\section{Une part croissante de diplômés non normaliens parmi les nouveaux enseignants}

En 2011, les diplômés des écoles normales n’ont représenté que $39 \%$ des presque 5300 nouveaux enseignants à Shanghai, et bien que cette part ait augmenté ces dernières années, elle n'excède toujours pas $50 \%$. Ce phénomène s'explique à deux niveaux :

\section{Renforcement du développement des personnels enseignants par l'État et évolutions de la structure industrielle du pays}

En 2011, la Chine a lancé la phase test de mise en œuvre de l'examen national du certificat d'enseignement ${ }^{5}$ ainsi qu'un programme de réforme pilote pour l'inscription régulière des enseignants des écoles primaires et secondaires, ce qui a contribué à élever le seuil d'accès à la profession enseignante et aboli la titularisation à vie de la qualification enseignante. De plus, en 2012, le gouvernement chinois a promulgué les Avis sur le renforcement du développement de la profession enseignante, premier document programmatique pour le développement global des personnels enseignants, de tous types et à tous les niveaux, qui prévoit la généralisation de la rémunération en fonction de la performance et l'augmentation graduelle du niveau de salaire des enseignants. Cet ensemble de mesures a créé des opportunités d'accès à la profession enseignante pour les diplômés non normaliens qui se dirigent vers l'éducation, mais également pour d'autres "talents» issus de carrières instables en raison des évolutions de la structure industrielle du pays.

\section{Insuffisance de la recherche en matière de formation initiale dans les écoles et universités normales et déclin de la compétitivité des étudiants normaliens}

Ces dernières années, le caractère spécifique des universités et écoles normales chinoises s'est affaibli suite à un processus interne de fusion, de modernisation et d'intégration. Comparées à ceux des pays développés, les heures de formation professionnelle en Chine n'ont représenté que $7 \%$ du total des heures de classe, soit une proportion significativement faible. En outre, ces heures de cours, déjà limitées et incluant une formation professionnelle insuffisante, se sont principalement concentrées sur l'étude théorique et ont négligé les aspects pratiques. Les étudiants normaliens reçoivent en général leur formation préprofessionnelle pendant leur période de stage, au cours de la dernière année, mais

5. National Teacher Certificate Examination en anglais. (NdT) 
un temps insuffisant lui est consacré et elle a souvent lieu dans des établissements d'accueil à faible niveau éducatif. De ce fait, un grand nombre d'étudiants normaliens ont de graves insuffisances en matière de compétences pédagogiques, ce qui vient annuler l'avantage compétitif lié à leur formation par rapport aux étudiants issus d'autres filières.

Finalement, l'insuffisance des expériences d'enseignement en stage et l'absence d'études systématiques en pédagogie, psychologie, méthodes d'enseignements, etc., fait qu'il est généralement difficile pour les nouveaux enseignants de s'adapter à leur poste d'enseignant, et encore plus aux tâches de gestion éducative.

\section{Les faiblesses du modèle de tutorat traditionnel}

Les nouveaux enseignants acquièrent traditionnellement leurs compétences pédagogiques auprès de leurs tuteurs désignés par leur école de recrutement, mais également par le biais de formations organisées par les instituts de formation des enseignants au niveau du district ou du comté pour la formation morale des enseignants et l'étude des théories éducatives. Les nouveaux enseignants ont également la possibilité de s'adapter rapidement à leur environnement de travail grâce à des mises en pratique répétées et à l'instruction directe de leurs tuteurs, eux-mêmes enseignants qualifiés dotés d'une expérience approfondie en matière de gestion des enseignants et dans les matières concernées.

Cependant, le problème de ce dispositif réside dans le fait que la qualité de la formation des nouveaux enseignants dépend souvent de l'instruction des tuteurs et de leur propre niveau d'enseignement, tandis que les résultats de cette formation ne sont souvent évalués que par le jugement subjectif des tuteurs. Ainsi, ce type de formation descendante par transmission d'expérience va en fait à l'encontre de l'amélioration globale du personnel enseignant débutant et de son futur développement professionnel.

De plus, avec l'accroissement démographique et l'expansion urbaine à Shanghai, la sélection des tuteurs devient de plus en plus difficile, notamment dans les écoles nouvellement construites et les écoles peu performantes des banlieues. Par conséquent, des enseignants presque débutants, avec seulement deux ou trois années d'expérience professionnelle, doivent eux-mêmes assumer la charge de tuteur, ce qui conduit de fait à une formation de niveau inégal et à une qualité non garantie.

\section{DES RÉFORMES DE LA FORMATION}

Ces dernières années, une importance croissante a été accordée au recrutement et à la promotion des nouveaux enseignants dans les provinces et villes chinoises. C'est par exemple le cas à Shanghai, qui met en œuvre un système de 
formation standardisée pour les enseignants en période de stage dans les écoles élémentaires et secondaires (maternelles incluses). Afin de résoudre les problèmes mentionnés précédemment et d'améliorer de manière globale la qualité professionnelle et la pratique pédagogique des enseignants stagiaires, Shanghai a mis en œuvre, en mars 2012, un programme de formation standardisée pour les enseignants stagiaires $(\mathrm{K}-12)^{6}$. Ainsi, pendant leur stage, les enseignants stagiaires reçoivent des enseignements aux contenus normés et selon des standards unifiés.

\section{Une normalisation de la méthode de formation}

La formation standardisée pour les nouveaux enseignants s'appuie sur les excellentes ressources éducatives présentes au niveau régional. Elle combine une formation centralisée dans les établissements de district, une formation en immersion dans les écoles de base, une formation en poste dans les écoles de recrutement et de l'auto-formation par les études. L'allègement des tâches enseignantes est ainsi associé à de la pratique pédagogique.

\section{La formation en immersion dans les écoles de base}

Il existe deux types d'écoles de base pour le développement professionnel des enseignants en Chine, respectivement au niveau des districts et des villes. Ces écoles ont généralement les qualifications suivantes : une longue tradition d'enseignement, une riche culture scolaire, un niveau de qualification global des enseignants élevé, une longue expérience et des résultats remarquables dans certaines matières, une excellente réputation d'enseignement, etc. Pendant leur période de stage, les nouveaux enseignants doivent passer la moitié de leur temps en immersion dans des écoles de base sélectionnées par le bureau municipal d'administration de l'éducation. Ils y apprennent la culture scolaire, encadrés par des groupes de recherche et des enseignants tuteurs d'excellence. Cela leur permet d'accumuler des ressources éducatives de grande qualité et de découvrir les perspectives de développement professionnel qui leur sont offertes; d'apprendre à accepter les conseils d'un tuteur à la formation et aux méthodes pédagogiques plus avancées et de prendre part à des activités de recherche et d'enseignement de haute qualité. Tout cela les aide à jeter les bases de leur futur projet de carrière et de développement professionnel.

Les tuteurs des écoles de base se rendent également dans les écoles où sont affectés les enseignants stagiaires pour observer leurs cours, les conseiller et travailler en lien avec les tuteurs des écoles de recrutement, afin d'optimiser

6. L'acronyme K-12 désigne en anglais les niveaux scolaires de la maternelle (Kindergarten) à la $12^{\mathrm{e}}$ année, correspondant à la fin du secondaire supérieur. $(N d T)$ 
l'impact de la formation des enseignants stagiaires. Grâce à la part importante accordée aux activités d'enseignement coopératives, mais également à leur fréquence et à leur intensité, ce mode de tutorat conjoint pourrait aider les enseignants stagiaires à affermir leur résolution et à élargir leurs visions pédagogiques.

\section{La formation en poste dans les écoles d'affectation}

La formation en poste de travail nécessite que les enseignants stagiaires s'engagent dans des activités d'enseignement dans les écoles de première affectation. Leurs activités d'enseignement et d'apprentissage dans les écoles de base et les autres établissements de formation feront partie intégrante de l'évaluation de leur performance.

\section{Une standardisation du contenu et des exigences de formation}

Le contenu de la formation standardisée pour les enseignants stagiaires se compose de quatre modules : motivation professionnelle et qualité morale ; expérience en classe et pratique d'enseignement (conception d'activités, d'enseignements et de soins adaptés aux enfants); gestion de classe et enseignement moral ; recherche éducative et développement professionnel. Ces quatre modules sont à leur tour divisés en 18 points clés (qui constituent 18 activités de formation), afin d'aider les enseignants stagiaires à développer des comportements pédagogiques adaptés, à améliorer leur aptitude à enseigner, à s'adapter le plus rapidement possible au rôle d'enseignant et à être compétents pour l'enseignement, de façon générale.

\section{Une standardisation de la gestion de la formation}

Pendant leur période de stage, les enseignants stagiaires signent un contrat avec leurs écoles de première affectation. Les enseignants stagiaires qualifiés recevront le "certificat de formation standardisé d'enseignant stagiaire de Shanghai $»^{7}$, délivré par le département d'éducation municipal et constituant la preuve de leur première inscription à la qualification enseignante. L'ensemble de ces mesures permet de garantir que les stagiaires suivent bien la formation. Dans le même temps, des équipes d'experts sont mises en place pour étudier, former, évaluer et faire des inspections annuelles dans les écoles de base au niveau des districts et des villes et dans les écoles de première affectation, afin de garantir le bon déroulement du processus de formation standardisée. 
À l'heure actuelle, le système de formation standardisée vise surtout à améliorer les cours de formation et à mener des recherches plus approfondies sur la formation des enseignants tuteurs. D’une part, en sélectionnant les cours de formation standardisée recommandés par les districts et les comtés et en aidant les concepteurs de cours à les adapter pour qu'ils soient interactifs, diversifiés et modulables en ligne, les cours de formation sont progressivement enrichis pour les stagiaires, et on espère que le conflit entre temps de travail et temps d'apprentissage pourra être résolu. D'autre part, les nouveaux enseignants qui participent au programme de formation standardisée et dont les performances sont remarquables servent de sujets de recherche à une étude empirique, dont l'objectif est de déterminer les facteurs du bon développement professionnel des enseignants débutants.

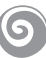

En dépit de ces réformes, il reste sans aucun doute de nombreux problèmes non résolus dans le système de formation initiale des enseignants en Chine. Par exemple, il demeure difficile pour les nouveaux enseignants de résoudre le conflit entre travail et apprentissage, et la formation standardisée des enseignants tuteurs devrait être plus largement prise en considération. Malgré le programme de formation standardisée mis en place, certaines écoles de première affectation n'ont pas réduit la charge de travail des nouveaux enseignants, pour des raisons liées au rendement du travail et à la pénurie de personnel. Les nouveaux enseignants sont harassés par la nécessité de devoir terminer leur formation et par le surmenage vécu dans les écoles de première affectation. Par ailleurs, les enseignants tuteurs délivrent trop d'idées et de messages à la fois, ce qui est susceptible de semer de la confusion chez les nouveaux enseignants. De leur côté, les enseignants tuteurs sont également soumis à une importante charge de travail sans être payés, ni formés en conséquence, et leur enthousiasme pédagogique s'en ressent. Les efforts et les recherches pour améliorer le programme de formation des enseignants doivent donc encore être poursuivis.

\section{BIBLIOGRAPHIE}

COMMISSION DE L'ÉDUCATION DE SHANGHAI (2012) : The Guidance on Standardized Training for Primary and Middle School (Kindergarten) Initial Teachers in Shanghai (Trial) [en ligne] [goo.gl/Jmr5sG] [en chinois] [consulté le 5 janvier 2017]. GOUVERNEMENT DE SHANGHAI (2010): Outline of Shanghai medium and long term education reform and development plan (2010-2020年) [en ligne] [goo.gl/0vOmc9] [en chinois] [consulté le 5 janvier 2017].

HU H.L. (2012) : Research on work guidance for initial teacher in America, mémoire de master, Université normale de Shanghai. 
LI M. (2013) : "Research on the influencing factors of satisfaction of primary and middle school initial teachers", Teacher Education Research, n 5, p. 43-48, Beijing Chinese (ISSN 1672-5905,CN 11-5147/G4), [en ligne, en chinois] [https://goo.gl/ $\mathrm{kjV} 3 \mathrm{Hs}]$

LIU C. Q. (2016) : Research on primary school initial teachers' occupational adaptability, mémoire de master, Université normale de Harbin.

YAN J., LIN Z. (2016) : "Initial teacher training and its enlightenment in Britain ", Educational Research, $\mathrm{n}^{\circ}$ 6, p. 148-155, Beijing Chinese (ISSN 1002-5731,CN 11-1281/G4), [en ligne, en chinois] [goo.gl/OBKdrD] 